\title{
Cellular and Molecular Biology of Metals
}

\author{
(Rudolfs K. Zalups and James Koropatnick (eds.), CRC Press, Taylor \& Francis Group, \\ Boca Raton-London-New York, 2010, 430 p., \$199.95)
}

DOI: $10.1134 /$ S0006297911040146

The book contains 14 chapters prepared by more than 30 international metal biology experts. The first chapter presents concepts and examples of cellular inorganic chemistry. There is consideration of the sources of metals, their molecular actions, metal-ligand binding, toxicity, metal-ion exchange chemistry, and common methodologies and assay systems.

Chapter 2 contains data about molecular and cellular biology of mercury in kidneys. The data of this chapter include transport of mercury in kidneys, urinary excretion of mercury, subcellular molecular interactions in renal epithelial cells, mercury and autoimmunity, and factors modifying the renal handling of mercury.

Chapter 3 highlights essential and toxic metal transport in the liver. The following aspects are discussed: models used to study hepatic transport, determinants of hepatic metal transport, peculiarities of various hepatic parts and organelles in metals uptake and release, transport across the canalicular membrane into bile and others topics of metals properties in the liver.

Chapter 4 consists of data about molecular and cell biology of lead. There is consideration of lead transport and cellular uptake in kidneys, cardiovascular system, immune and reproductive systems, in developing of renal cancer. Two of the last parts of this chapter focus on pharmacological consideration and $\mathrm{Pb}$-based nanomaterials.

Chapter 5 describes transport and biological impact of manganese. There is discussion on manganese transport in lungs and brain and molecular mechanism of abnormal Mn levels.

Chapter 6 focuses on metallothionein (MT) and metal homeostasis. There is analysis of MT proteins, gene structure and function, MT in health and disease, including neurological function.

Chapter 7 is devoted to the cellular and molecular biology of iron-binding proteins. There are data about iron metabolism and transport and also iron homeostasis including regulation by the hormone hepcidin.

In the chapter 8 there is discussion on regulatory and signaling functions of zinc ions in human cellular physiology. There is a special part of this chapter dealing with specialized cells that secrete zinc.

Chapter 9 highlights glutathione, protein thiols, and metal homeostasis.

Chapter 10 is devoted to ionic and molecular mimicry and the transport of metals. The data related to arsenic, cadmium, lead, mercury, selenium, and such toxic metal forms as arsenate, chromate, molybdate, and vanadate.

Chapter 11 highlights heavy metal transport and detoxification in crustacean gastrointestinal and renal epithelial cells.

Chapter 12 deals with metals and cell adhesion molecules. There is overview of cell adhesion and inflammatory adhesion molecules, including integrins, cadherin/ catenin complexes and discussion of potential mechanisms underlying the effects of metals on these molecules.

Chapter 13 is devoted to iron metabolism and disease. There is discussion of physiology and hormonal control of iron homeostasis and traffic, iron deficiency and overload, hereditary hemochromatosis, ferroportin disease, hyperferritinemia-cataract syndrome, and other pathological conditions due to abnormality in iron metabolism.

Chapter 14 highlights the influences of metals on immune function. There is consideration of metal effect on immune mechanisms, immunotoxic effect of nonessential metals, and metal-mediated immunosuppression and immunomodulation.

Each chapter contains a basic bibliography related to book topics and subject index at the end of the book. The book will be useful for researchers in biochemistry, molecular biologists and physicians, and teachers and students of medical schools and universities specialized in cellular and molecular biology of metals. 